\title{
Acknowledgement to Reviewers of Laws in 2018
}

\author{
Laws Editorial Office
}

MDPI, St. Alban-Anlage 66, 4052 Basel, Switzerland

Published: 9 January 2019

\begin{abstract}
Rigorous peer-review is the corner-stone of high-quality academic publishing. The editorial team greatly appreciates the reviewers who contributed their knowledge and expertise to the journal's editorial process over the past 12 months. In 2018, a total of 35 papers were published in the journal, with a median time to first decision of 32 days and a median time to publication of 75 days. The editors would like to express their sincere gratitude to the following reviewers for their cooperation and dedication in 2018:
\end{abstract}

Avi-Yonah, Reuven
Bartlett, Peter
Baumgarth, William P.
Bombard, Yvonne
Bracci, Enrico
Bridge, Dave
Brolan, Claire E.
Burić, Zoran
Capannini-Kelemen, Katalin
Carney, Terry
Cerchia, Rossella Esther
Christensen, Keith
Conway, Gerard
Cosentino, Antonietta
Dickens, Bernard
Diesfeld, Kate
Dimopoulos, Andreas
Dinerstein, Robert
Dixon, Rosalind
Fabri, Marco
Francis, Leslie
Franklin, Aimee
Frost, Tom
Frymer, Paul
Gavrielides, Theo
Geist, Michael
Gibbons, Llewellyn
Gleason, Shane A.
Gooding, Piers
Greggi, Marco
Guns, Wendy
Higgins, Noelle
Hinkle, Rachael K.
Ismer, Roland
Joly, Yann

Kim, JaeWon

Knowles, Helen J.

Kupferman, Scott

Kurrild-Klitgaard, Peter

Kyriakopoulos, Grigorios

Lenz, Timothy

Liu, Yigong

Luchenitser, Alex J.

Mac Síthigh, Daithí

Magu, Stephen

Marshall, Virginia

Melear, K. B.

Miller, Mark C.

Mulroy, Quinn

Nava, Luzma Fabiola

Nelson, Michael

Noked, Noam

Nolette, Paul

Olivas, Raul Navarro

Percival, Robert

Ruda González, Albert

Ryan, Christopher James

Scholten, Matthé

Selin, Jennifer

Senter, Leigha

Series, Lucy

Seymour, Kate

Simeon, James C.

Siobhan, Weare

Sjöström, Stefan

Slobogin, Christopher

Sołoducho-Pelc, Letycja M.

Spagnoli, Paola

Spijkers, Otto

Staszak, Sarah 
Stavert, Jill

Su, Yi-Yuan

Sugg, Zachary P.

Tepperová, Jana

Tranter, Kieran

Turenne, Sophie Vakilifathi, Mona
Varady, Robert

Vera Gray, Fiona

Walters, Daniel

Wasileski, Gabriela

West, Raelene

Yoon, SunMan

Zelle, Heather

(C) 2019 by the author. Licensee MDPI, Basel, Switzerland. This article is an open access article distributed under the terms and conditions of the Creative Commons Attribution (CC BY) license (http://creativecommons.org/licenses/by/4.0/). 